\title{
DETERMINAÇÃO DO “STATUS” NUTRICIONAL DE NITROGÊNIO NO FEIJOEIRO UTILIZANDO IMAGENS DIGITAIS COLORIDAS ${ }^{1}$
}

\author{
MURILO M. BAESSO ${ }^{2}$, FRANCISCO DE A. DE C. PINTO ${ }^{2}$, DANIEL M. DE QUEIROZ ${ }^{3}$, \\ LUCIANO B. DE VIEIRA ${ }^{3}$, ENRIQUE A. ALVES ${ }^{2}$
}

RESUMO: Este trabalho teve como objetivo avaliar o uso de índices espectrais, retirados de imagens digitais, para discriminar diferentes doses de $\mathrm{N}$ no feijoeiro. $\mathrm{O}$ trabalho, conduzido em vasos de $8 \mathrm{dm}^{3}$, teve cinco tratamentos $\left(0 ; 50 ; 100 ; 150\right.$ e $\left.200 \mathrm{~kg} \mathrm{de} \mathrm{N} \mathrm{ha}^{-1}\right)$, com dez repetições. As imagens foram adquiridas aos 30; 40 e 50 dias após a emergência. Foram desenvolvidas funções discriminantes quadráticas, tendo como vetores de entrada as médias dos "pixels" de diferentes combinações dos quatro índices espectrais testados. Três diferentes tamanhos de blocos de imagem foram testados $9 \times 9 ; 20 \times 20$ e 40 × 40 "pixels". Os melhores resultados foram alcançados pelos blocos de 9 x 9 e 20 x 20 "pixels", apresentando classificação 94; 96 e 96\% superior à classificação ao acaso para os blocos 9 x 9 "pixels" e 92; 94 e 94\% para os blocos 20x20 "pixels" aos 30; 40 e 50 dias após a emergência, respectivamente.

PALAVRAS-CHAVE: agricultura de precisão, processamento de imagens digitais, classificador estatístico.

\section{DETERMINATION OF NITROGEN STATUS IN BEAN PLANTS USING COLOR DIGITAL IMAGES}

ABSTRACT: The objective of this work was to evaluate image spectral indices to detect nitrogen levels applied to bean plants. The experiment was in pots with $8 \mathrm{dm}^{3}$ of soil, five treatments $(0 ; 50$; $100 ; 150$ and $200 \mathrm{~kg}$ of $\mathrm{N} \mathrm{ha}^{-1}$ ) with ten repetitions. The images were taken at 30; 40 and 50 days after emergence. Quadratic discriminant functions were developed, and the input vector was the average of the pixels from different combinations of four spectral indices. Three different sizes of image blocks were tested: $9 \times 9,20 \times 20$ and $40 \times 40$ pixels. The best results were obtained when using the $9 \times 9$ and $20 \times 20$ pixels blocks, with classification performance of 94; 96 e $96 \%$ better than a random classification for the blocks $9 \times 9$ pixels and 92; 94 and 94\% for the blocks $20 \times 20$ pixels at 30; 40 e 50 days after emergency, respectively.

KEYWORDS: precision farming, digital image processing, statistical classification.

\section{INTRODUÇÃO}

Na cultura do feijoeiro, o nitrogênio é o macronutriente mais absorvido (ROSOLEM \& MARUBAYASHI, 1994), estimulando o crescimento vegetativo da planta. Em aplicações convencionais de fertilizantes, toda a área de um talhão é considerada homogênea, ou seja, a necessidade de adubo é tida como igual para toda a área, o que na realidade não ocorre. Baseandose nas necessidades médias da planta, uma aplicação uniforme causa adubação excessiva em algumas áreas do campo e insuficiente em outras, com comprometimento da eficiência do processo de produção. Nesses casos, a agricultura de precisão é uma alternativa ao sistema convencional, com aplicação de nutrientes em doses variadas.

Uma maneira prática e rápida de determinar a existência da deficiência de nitrogênio na planta é por meio do diagnóstico visual. Porém, a sua precisão está limitada à experiência do

\footnotetext{
${ }^{1}$ Extraído da dissertação de Mestrado do primeiro autor.

${ }^{2}$ Engo Agrônomo, Doutorando em Engenharia Agrícola, Universidade Federal de Viçosa, Viçosa - MG, murilo@ vicosa.ufv.br

${ }^{3}$ Eng $^{\mathrm{o}}$ Agrícola, Professor Adjunto, Departamento de Engenharia Agrícola, Viçosa - MG, Fone: (0XX31) 3899-1881, facpinto@ufv.br, Bolsista do CNPq.

Recebido pelo Conselho Editorial em: 25-4-2005

Aprovado pelo Conselho Editorial em: 4-4-2007
} 
técnico e, também, exige bastante trabalho para gerar um mapa de prescrição para ser utilizado no manejo localizado. Visualmente, o sintoma da deficiência de $\mathrm{N}$ é uma clorose nas folhas, que tem início nas mais velhas, o que possibilita o uso das propriedades espectrais do dossel da cultura para detectar essa deficiência.

Diversas pesquisas vêm demonstrando o potencial da utilização das propriedades espectrais para identificar a variabilidade espacial do "status" de nitrogênio em culturas agrícolas (BLACKMER et al., 1996; SÉRÉLÉ et al., 2000; HABOUDANE et al., 2002; VARELLA et al., 2005). Outras pesquisas demonstraram o potencial dessas propriedades também para a cultura do feijoeiro (FURLANI JÚNIOR et al., 1996; SILVEIRA et al., 2003), porém correlacionando medidas de clorofila com o "status" do nitrogênio, o que, no contexto da agricultura de precisão, exigiria grande número de medições para a caracterização da variabilidade espacial do "status" de nitrogênio. Essa taxa de amostragem exigida no manejo localizado poderia ser alcançada com a utilização de técnicas de sensoriamento remoto. Dessa maneira, a hipótese da presente pesquisa é que se pode identificar o "status" nutricional do nitrogênio no feijoeiro por meio de processamento e análise de imagens digitais.

Este trabalho teve como objetivo avaliar o uso de índices espectrais, obtidos a partir de imagens digitais, para discriminar feijoeiros submetidos a diferentes doses de $\mathrm{N}$.

\section{MATERIAL E MÉTODOS}

O trabalho foi conduzido em casa de vegetação, na cidade de Viçosa - MG, localizada à altitude de aproximadamente $650 \mathrm{~m}$, com coordenadas geográficas de $20^{\circ} 45^{\prime} 54^{\prime \prime}$ ' latitude sul e 45 52'54' ' longitude oeste. O trabalho teve cinco tratamentos $\left(0 ; 50 ; 100 ; 150\right.$ e $\left.200 \mathrm{~kg} \mathrm{de} \mathrm{N} \mathrm{ha}^{-1}\right)$, com dez repetições, totalizando 50 vasos com $8 \mathrm{dm}^{3}$ de material de solo retirado do horizonte $\mathrm{C}$ de um solo classificado como Latossolo Vermelho distrófico.

O cultivo foi realizado em vasos para que houvesse melhor controle das quantidades aplicadas de nutrientes. Foram semeadas cinco sementes de feijão (Phaseolus vulgaris L.) da cultivar BRSMG Talismã por vaso. Aos 20 dias após a emergência (DAE), procedeu-se ao desbaste, deixando-se três plantas por vaso. Como o nitrogênio é um nutriente de alta mobilidade no solo, a dose foi aplicada em duas etapas: um terço do total no ato do plantio e o restante aos 20 DAE. Os demais nutrientes foram misturados de maneira uniforme em todos os vasos com o material de solo, de acordo com os resultados da análise de solo, ficando apenas o nitrogênio como limitante na produção do feijão. Foi feita uma irrigação diária para que não houvesse nenhuma influência do déficit hídrico no desenvolvimento das plantas. Para a validação do nível de deficiência de nitrogênio na planta, foram feitas análises foliares em todos os vasos de cada tratamento aos 50 DAE.

Para a obtenção das imagens, foi utilizada uma câmera colorida digital da marca JAI, modelo CV-M7+CL, padrão CamLink ${ }^{\circledR}$, ligada a um microcomputador pessoal. A câmera possui CCD de 2/3" da marca Sony, modelo ICX285AQ, cuja sensibilidade espectral é apresentada na Figura 1. A interface entre o microcomputador e a câmera foi realizada por uma placa de aquisição de vídeo da marca Coreco Imaging, modelo PC-CamLink ${ }^{\circledR}$. As imagens foram armazenadas no formato TIFF ("tagged image file format") com dimensão de 1.380 (h) x 1.030 (v) "pixels", sendo utilizada lente da marca Tamron, modelo 23FM25L, com distância focal de $25 \mathrm{~mm}$.

Para que as imagens fossem obtidas em condições de iluminação controlada, foi construída uma estrutura metálica com dimensões de 1,50 x 1,50 m e 2,00 m de altura, recoberta com tecido do tipo "blackout". O sistema de iluminação era composto por oito refletores com lâmpadas halógenas de $150 \mathrm{~W}$, direcionadas para cima, para que as plantas não recebessem luz direta. A câmera foi posicionada a $2 \mathrm{~m}$ acima da planta, adquirindo imagem com resolução espacial de $0,27 \mathrm{~mm}_{\text {pixel }}{ }^{-1}$.

Com o objetivo de avaliar a melhor época para a obtenção das imagens, essas foram feitas em três épocas: 30; 40 e 50 DAE. As medições aos 50 DAE foram realizadas após identificar que 
poucas plantas estavam no estádio inicial de floração, ou seja, a maioria ainda não tinha apresentado sinal de pré-senescência natural, e que alguns autores recomendam a última dose nitrogenada, quando parcelada, entre 45 e 50 DAE (ARF et al., 1999; FAHL et al., 1998).

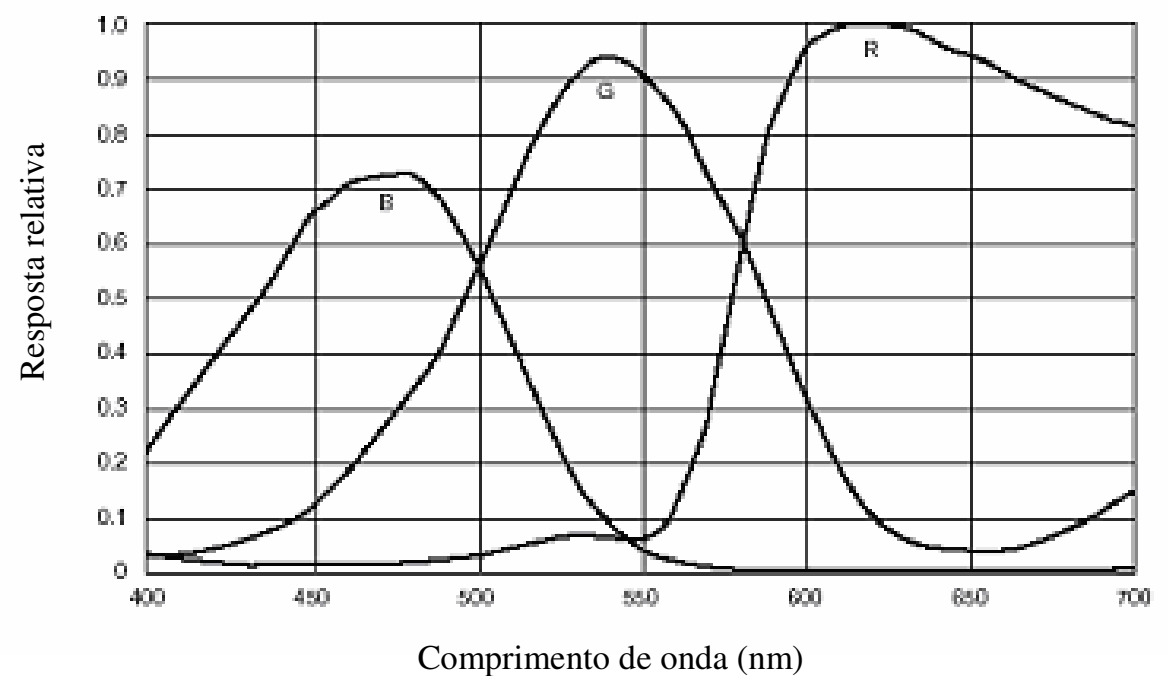

FIGURA 1. Curvas da sensibilidade espectral da câmera digital da marca JAI, modelo CVM7+CL. (Fonte: Manual Sony CCD ICX285AQ).

O valor digital do "pixel" é função do sistema óptico (câmera e lente), da iluminação, da posição relativa planta-sensor-iluminação e das propriedades espectrais da planta. Tendo em vista que o sistema óptico, a iluminação e a posição relativa planta-sensor-iluminação foram controlados e mantiveram-se constantes durante as medições, as variações do valor digital do "pixel" foram assumidas serem somente devidas às variações das propriedades espectrais da planta. Em situações práticas, em que a iluminação não pode ser controlada, deve-se acrescentar ao processamento algumas técnicas de normalização do valor digital para que o mesmo esteja apenas relacionado com as propriedades espectrais da planta. Isso poderia ser obtido com a medição da radiação incidente ou, até mesmo, a utilização da própria planta como padrão, conforme proposto por BLACKMER et al. (1996). O presente trabalho não teve como objetivo desenvolver metodologia de normalização do valor digital do "pixel", utilizando, dessa maneira, iluminação controlada.

As imagens foram processadas no Laboratório de Projeto de Máquinas e Visão Artificial (PROVISAGRO) do Departamento de Engenharia Agrícola da Universidade Federal de Viçosa. Foi utilizado o sistema computacional MATLAB ${ }^{\circledR} 6.0$ com o pacote de ferramentas para processamento de imagens. Em vez de utilizar a imagem original, utilizou-se um vetor com características que representam a informação desejada. Este trabalho partiu da hipótese de que quatro índices espectrais portam a informação desejada para classificar o "status" nutricional do nitrogênio na planta. Dessa maneira, o processamento de imagens visou à geração de um vetor de características cujos elementos eram índices espectrais.

Os índices espectrais utilizados como características para classificar o "status" nutricional do feijoeiro foram:

$$
\begin{aligned}
& \mathrm{EVd}=\frac{2 \mathrm{Vd}-\mathrm{Vm}-\mathrm{Az}}{\mathrm{Vm}+\mathrm{Vd}+\mathrm{Az}} \\
& \text { Vern }=\frac{\mathrm{Vm}}{\mathrm{Vm}+\mathrm{Vd}+\mathrm{Az}} \\
& \mathrm{Vn}=\frac{\mathrm{Vd}}{\mathrm{Vm}+\mathrm{Vd}+\mathrm{Az}} \\
& \mathrm{Rvv}=\frac{\mathrm{Vd}}{\mathrm{Vm}}
\end{aligned}
$$


em que,

Evd - excesso de verde;

Vd - média dos "pixels" na banda do verde;

Az - média dos "pixels" na banda do azul;

Vm - média dos "pixels" na banda do vermelho;

Vern - vermelho normalizado;

Vn - verde normalizado, e

Rvv - razão verde-vermelho.

As propriedades espectrais dos pigmentos das plantas, principalmente a absorção da clorofila na região do espectro vermelho e/ou reflectância na região do verde vêm sendo exploradas em conjunto com a reflectância do infravermelho próximo para a estimativa do "status" de nitrogênio das plantas (MOGES et al., 2004; SCHRODER et al., 2000; GITELSON et al., 1996), devido a clorofila estar positivamente associada com concentração de N (WOLFE et al., 1988). Nesses trabalhos, utilizam-se os índices da diferença normalizada com a banda vermelha (NDVI) ou com a banda verde (GNDVI). Nos trabalhos desenvolvidos por GITELSON et al. (1996) e SENA JÚNIOR (2005), a banda verde apresentou-se mais eficiente para discriminar entre os diferentes "status" de N. Tendo em vista o custo mais elevado das câmeras para aquisição da banda do infravermelho próximo, no presente trabalho, testaram-se apenas índices com as bandas do visível.

$\mathrm{O}$ índice excesso de verde $(\mathrm{EVd})$ vem sendo utilizado principalmente para facilitar a discriminação entre material verde do solo (ORLANDO et al., 2004). No presente trabalho, partiuse da hipótese de que o realce da informação na banda verde poderia melhorar o poder de discriminação entre os diferentes "status" de N. Também se testou a utilização de apenas uma banda (Vm e Vern) para a discriminação entre os diferentes "status" nutricionais, possibilitando futuramente a utilização de câmera monocromática com filtro óptico. Por último, utilizou-se a combinação das duas bandas de maior relação com as propriedades espectrais das plantas no visível (Rvv).

Todas as características testadas (EVd, Vm, Vern e Rvv) foram normalizadas para minimizar o efeito da variação da iluminação no experimento.

Essas características foram calculadas para blocos da imagem os quais foram assumidos, correlacionados às propriedades espectrais da folha. Neste trabalho, foram testados blocos da imagem com dimensões de 9 × 9; 20 x 20 e 40 x 40 "pixels". De cada imagem de um vaso recortaram-se aleatoriamente cinco blocos, e a média dos valores de "pixels" desses blocos representou uma observação do vetor de característica.

O "status" nutricional do feijoeiro foi identificado com base na classificação do vetor de características. Foram testados 15 diferentes vetores de características: quatro com os índices isoladamente, seis com combinações de dois índices, quatro com combinações de três índices e um com os quatro índices. No presente trabalho, foi testado um classificador estatístico, descrito por GONZALES \& WOODS (1992). Considerando a mesma probabilidade a priori de ocorrência das cinco doses de adubação de nitrogênio, o classificador era composto por um conjunto de funções discriminantes:

$$
\mathrm{d}_{\mathrm{j}}(\mathrm{X})=-\frac{1}{2} \ln (\operatorname{det} \mathrm{j})-\frac{1}{2}\left[\left(X-\mathrm{m}_{\mathrm{j}}\right)^{\mathrm{T}} \mathrm{C}_{\mathrm{j}}^{-1}\left(\mathrm{X}-\mathrm{m}_{\mathrm{j}}\right)\right]
$$

em que,

$\mathrm{J}$ - classe de estresse nutricional, 1 a 5 ;

$\mathrm{d}_{\mathrm{j}}$ - função discriminante da classe $\mathrm{j}$;

$\mathrm{C}_{\mathrm{j}}$ - matriz de covariância da classe $\mathrm{j}$;

$\operatorname{det} C_{j}$ - determinante da matriz de co-variância da classe $j$;

$\mathrm{X}$ - vetor de características;

$\mathrm{m}_{\mathrm{j}}-$ vetor de características médio da classe $\mathrm{j}$, e

$\mathrm{T}$ - símbolo que representa a transposta de uma matriz. 
Foram desenvolvidas cinco funções discriminantes, uma para cada classe de estresse nutricional, de forma que um vetor desconhecido $\mathrm{X}$ foi atribuído à classe $\mathrm{j}$, que apresentou o maior valor da função discriminante $\mathrm{d}_{\mathrm{j}}(\mathrm{X})$. $\mathrm{O}$ classificador estatístico também foi utilizado para a definição do melhor vetor de características. $\mathrm{O}$ erro de classificação foi estimado por validação cruzada, deixando uma observação de fora (KHATTREE \& NAIK, 2000). Nesse processo, uma observação foi separada, e as funções discriminantes foram elaboradas com o restante dos dados (49 amostras), e, em seguida, a observação separada foi classificada. Esse procedimento foi repetido sucessivamente para cada amostra, ou seja, todas as 50 observações (cinco tratamentos e dez repetições). A seguir, foi elaborada uma matriz de erro, conforme descrito em CONGALTON (1991).

A avaliação do desempenho dos classificadores foi determinada pelo coeficiente Kappa. $\mathrm{O}$ coeficiente Kappa foi estimado pela seguinte equação:

$$
\hat{\mathrm{K}}=\frac{\mathrm{n}_{\mathrm{t}} \sum_{\mathrm{i}=1}^{\mathrm{c}} \mathrm{x}_{\mathrm{ii}}-\sum_{\mathrm{i}=1}^{\mathrm{c}} \mathrm{x}_{\mathrm{i} \oplus} \mathrm{x}_{\oplus \mathrm{i}}}{\mathrm{n}_{\mathrm{t}}{ }^{2}-\sum_{\mathrm{i}=1}^{\mathrm{c}} \mathrm{x}_{\mathrm{i} \oplus} \mathrm{x}_{\oplus \mathrm{i}}}
$$

em que,

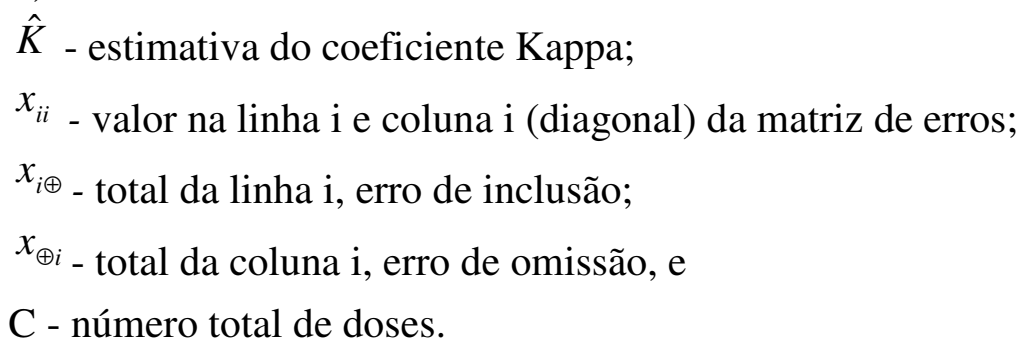

Por fim, para definir os melhores classificadores, foi feito o teste $\mathrm{Z}$ a $5 \%$ para testar a diferença entre dois coeficientes Kappa (CONGALTON \& MEAD, 1983). A variância do coeficiente Kappa foi estimada conforme BISHOP et al. (1975), citados por HUDSON \& RAMM (1987).

\section{RESULTADOS E DISCUSSÃO}

$\mathrm{Na}$ análise de variância para as médias dos valores de análises foliares aos 50 DAE, observou-se que os efeitos de doses foram significativos $(\mathrm{P}<0,0001)$. Portanto, a procura de um classificador para discriminar os diferentes níveis de N pode ser justificada (REGAZZI, 2000).

Não houve diferença significativa entre os tratamentos 50; 100 e $150 \mathrm{~kg}$ de $\mathrm{N} \mathrm{ha}^{-1}$ (Tabela 1). Sendo assim, o classificador estatístico foi desenvolvido apenas para três classes de "status" nutricional de nitrogênio, sendo elas: $0 ; 200 \mathrm{~kg} \mathrm{de} \mathrm{N} \mathrm{ha}^{-1}$ e outra englobando as três classes intermediárias $\left(50 ; 100\right.$ e $150 \mathrm{~kg}$ de $\mathrm{N} \mathrm{ha}^{-1}$ ). Nas Figuras 2 a 4, são apresentados exemplos de imagens utilizadas neste trabalho para os 30; 40 e 50 dias após a emergência, respectivamente. Em cada figura, também são destacados os blocos recortados com 9 x 9; 20 x 20 e 40 x 40 "pixels" utilizados no desenvolvimento dos classificadores estatísticos.

TABELA 1. Médias das análises foliares para as doses de N, realizada aos 50 DAE.

\begin{tabular}{cc}
\hline $\mathrm{kg} \mathrm{de} \mathrm{N} \mathrm{ha}$ & Média dos Valores de Análise Foliar $\left(\mathrm{g} \mathrm{kg}^{-1}\right)$ \\
\hline 200 & $51,6 \mathrm{a}$ \\
150 & $44,7 \mathrm{~b}$ \\
100 & $41,8 \mathrm{~b}$ \\
50 & $39,5 \mathrm{~b}$ \\
0 & $31,8 \mathrm{c}$ \\
\hline
\end{tabular}

Valores seguidos de uma mesma letra não diferem, na coluna, pelo teste Duncan, a 5\% de probabilidade. 

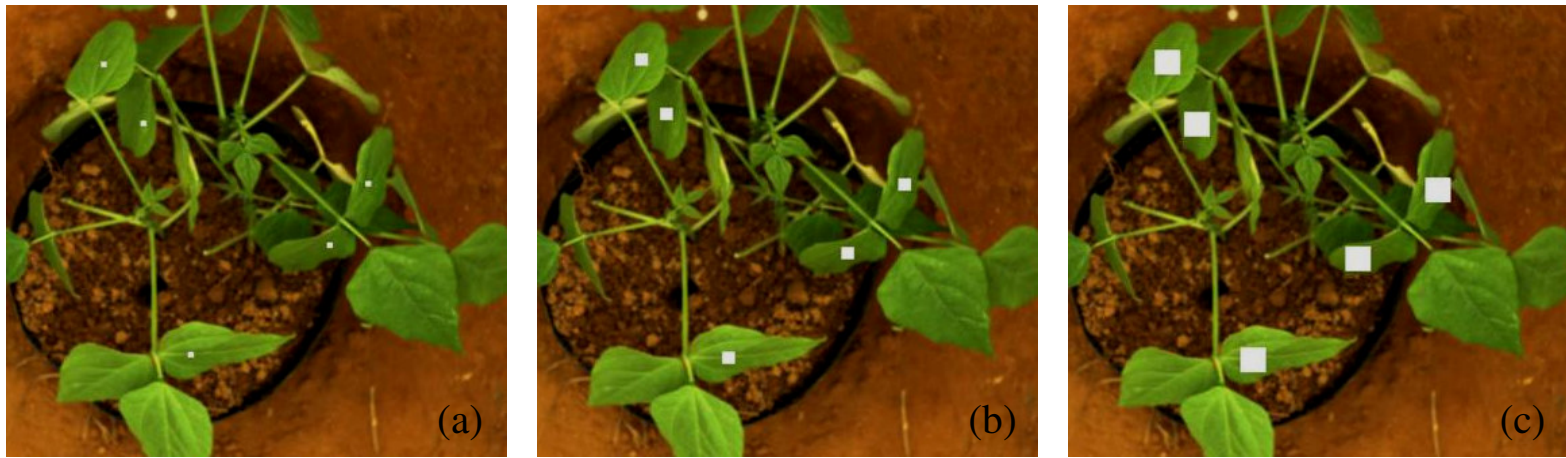

FIGURA 2. Exemplo de imagens utilizadas neste trabalho, adquiridas aos 30 dias após a emergência, com destaque em branco para os recortes de blocos com (a) 9 x 9; (b) 20 x 20 e (c) 40 x 40 "pixels".
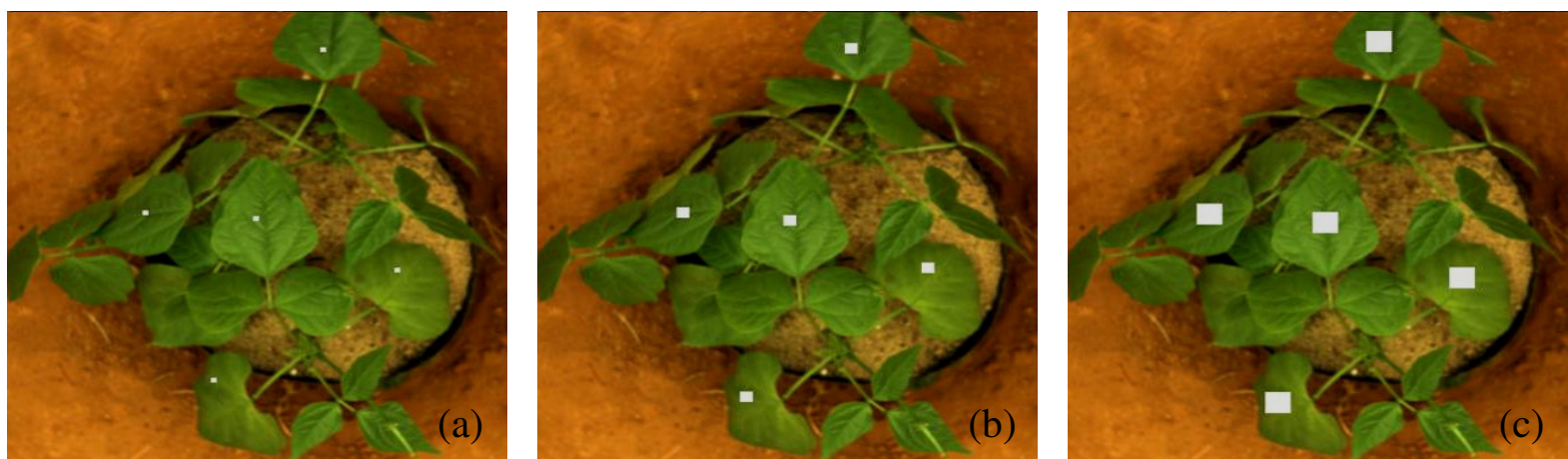

FIGURA 3. Exemplo de imagens utilizadas neste trabalho, adquiridas aos 40 dias após a emergência, com destaque em branco para os recortes de blocos com (a) 9 x 9; (b) 20 x 20 e (c) 40 x 40 "pixels".
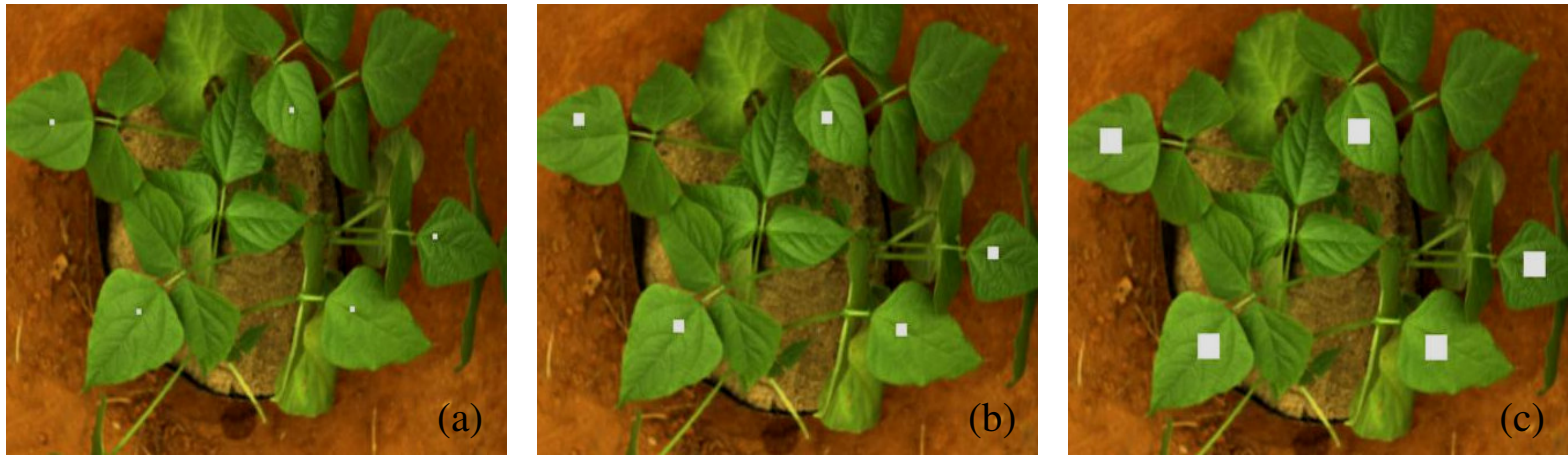

FIGURA 4. Exemplo de imagens utilizadas neste trabalho, adquiridas aos 50 dias após a emergência, com destaque em branco para os recortes de blocos com (a) 9 x 9; (b) 20 x 20 e (c) 40 x 40 "pixels".

Segundo os critérios sugeridos por LANDIS \& KOCH (1977), citados por ADAMI et al. (2002), o classificador é tido como excelente quando apresenta valor de Kappa igual ou superior a 0,8. Apesar de esse valor ser arbitrário e ter sido sugerido para fins de diagnóstico clínico, conforme destacam os autores, esse valor de Kappa de 0,8 foi adotado no presente trabalho como limiar abaixo do qual se descartaram os classificadores. Sendo assim, na Tabela 2, são apresentadas as frequiências de ocorrência de classificadores com Kappa igual ou superior a 0,8 para cada época de aquisição de imagens e para cada combinação de índices espectrais. 
TABELA 2. Freqüências de ocorrência de classificadores com Kappa igual ou superior a 0,8 para cada combinação de índices espectrais utilizados como vetor de características.

\begin{tabular}{cc}
\hline Índices Espectrais & Total* \\
\hline Evd & 4 \\
Vern & 0 \\
Vn & 4 \\
Rvv & 5 \\
Evd e Vern & 7 \\
Evd e Vn & 2 \\
Evd e Rvv & 8 \\
Vern e Vn & 7 \\
Vern e Rvv & 6 \\
Rvv e Vn & 8 \\
Evd, Vern e Vn & 6 \\
Evd, Vern e Rvv & 6 \\
Evd, Vn e Rvv & 6 \\
Vern, Vn e Rvv & 6 \\
Evd, Vern, Vn e Rvv & 6 \\
Total & 81
\end{tabular}

Evd - excesso de verde; Vern - vermelho normalizado; Vn - verde normalizado; Rvv - razão verde-vermelho. * Frequiência de ocorrência máxima igual a 9 (três épocas x três tamanhos de blocos)

Os melhores desempenhos normalmente ocorreram com a utilização de mais de um índice espectral no vetor de características do classificador. Dessa maneira, apesar da sobreposição da sensibilidade da câmera nas diferentes bandas (Figura 1), devido à baixa resolução espectral da mesma, a utilização de mais de um índice espectral facilitou a discriminação das diferentes doses de $\mathrm{N}$ utilizadas neste trabalho. A utilização de câmeras com faixas de sobreposição menores, ou seja, com sensibilidade concentrada nas bandas de interesse $(550 \mathrm{~nm}$ e $650 \mathrm{~nm})$, pode realçar as propriedades espectrais das plantas, melhorando a discriminação entre "status" de N. Os coeficientes Kappa dos melhores resultados de classificação desenvolvidos aos 30; 40 e 50 DAE para os blocos de 9 x 9; 20 x 20 e 40 x 40 “pixels" são apresentados na Tabela 3.

TABELA 3. Coeficientes Kappa dos melhores resultados de classificação para os blocos de 9 x 9; 20 x 20 e 40 x 40 "pixels" das imagens obtidas aos 30; 40 e 50 DAE.

\begin{tabular}{cccc}
\hline Bloco & \multicolumn{3}{c}{ Época } \\
\cline { 2 - 4 } & $30 \mathrm{DAE}$ & $40 \mathrm{DAE}$ & $50 \mathrm{DAE}$ \\
\hline 9 x 9 "pixels" & $0,94 \mathrm{Aa}$ & $0,96 \mathrm{Aa}$ & $0,96 \mathrm{Aa}$ \\
20 x 20 "pixels" & $0,92 \mathrm{Aa}$ & $0,94 \mathrm{Aa}$ & $0,94 \mathrm{Aa}$ \\
40 x 40 "pixels" & $0,82 \mathrm{Aa}$ & $0,82 \mathrm{Aa}$ & $0,76 \mathrm{Ba}$ \\
\hline
\end{tabular}

ns - Não diferem de zero pelo teste Z, a 5\% de probabilidade; os coeficientes Kappa seguidos de mesma letra minúscula não diferem na linha pelo teste $Z$, a $5 \%$ de probabilidade; os coeficientes Kappa seguidos de uma mesma letra maiúscula não diferem, na coluna, pelo teste $\mathrm{Z}$, a $5 \%$ de probabilidade.

Os resultados observados na Tabela 3 sugerem que o tamanho de bloco não influenciou na classificação do "status" de nitrogênio estudado neste trabalho; sendo assim, tanto os blocos 20 x 20 "pixels" $\left(29,16 \mathrm{~mm}^{2}\right)$ quanto os de 9 x 9 "pixels" $\left(5,90 \mathrm{~mm}^{2}\right)$ são os recomendados para a utilização em sistema de discriminação das doses de $\mathrm{N}$ estudadas, já que foram estatisticamente melhores que os blocos 40 x 40 pixels $\left(116,64 \mathrm{~mm}^{2}\right)$, aos 50 DAE.

\section{CONCLUSÕES}

O classificador estatístico foi capaz de avaliar o status do N no feijoeiro. 
Os melhores desempenhos de classificação ocorreram com a utilização de mais de um índice espectral no vetor de características do classificador.

O tamanho de bloco não influenciou na classificação do "status" de nitrogênio estudado neste trabalho.

\section{REFERÊNCIAS}

ADAMI, M.; PINHEIRO, E.S.; MOREIRA, M.A. Aplicação de diferentes algoritmos para a classificação de imagens ETM+/Landsat-7 no mapeamento agrícola. In: SIMPÓSIO LATINOAMERICANO DE PERCEPCIÓN REMOTA Y SISTEMAS DE INFORMACIÓN ESPACIAL, 10., 2002, Cochabamba. Anais... 2002. 1 CD-ROM.

ARF, O.; FERREIRA, E.C.; CARVALHO, M.A.C.; SÁ, M.E.; BUZETTI, S. Efeito de doses e parcelamento da adubação nitrogenada em cobertura na cultura do feijão. In: REUNIÃO NACIONAL DE PESQUISA DE FEIJÃO, 6., 1999, Salvador. Resumos... Goiânia: Embrapa, 1999. p.790-3.

BLACKMER, T.M.; SCHEPERS, J.S.; VARVEL, G.E.; MEYER, G.E.. Analysis of aerial photography for nitrogen stress within corn fields. Agronomy Journal, Madison, v.88, n.5, p.72933, 1996.

CONGALTON, R.G. A review of assessing the accuracy of classifications of remotely sensed data. Remote Sensing Thematic Accuracy Assessment: A compendium, New York, v.37, n.1, p.35-46, 1991.

CONGALTON, R.G.; MEAD, R.A. A quantitative method to test for consistency and correctness in photo interpretation. Photogrammetric Engineering \& Remote Sensing, Falls Church, v.49, n.1, p.69-74, 1983.

FAHL, J.I.; CAMARGO, M.B.P.; PIZZINATTO, M.A.; BETTI, J.A.; MELO, A.M.T.; DEMARIA, I.C.; FURLANI, A.M.C. Instruções agrícolas para as principais culturas econômicas. Campinas: Instituto Agronômico de Campinas, 1998. v.1, p.396.

FURLANI JÚNIOR, E.; NAKAGAWA, J.; BULHÕES, L.J.; MOREIRA, J.A.A.; GRASSI FILHO, H. Correlação entre leituras de clorofila e níveis de nitrogênio aplicados em feijoeiro. Bragantia, Campinas, v.55, n.1, p. 171-5, 1996.

GITELSON, A.A.; KAUFMAN, Y.J.; MERZYAK, M.N. Use of a green channel in remote sensing of global vegetation from EOS-MODIS. Remote Sensing of Environment, New York, v.58, n.3, p.289-98, 1996.

GONZALEZ, R.C.; WOODS, R.E. Digital image processing. Reading Massachusetts: AddisonWesley Publishing Company, 1992, 716p.

HABOUDANE, D.; MILLER, J.R.; TREMBLAY, N.; ZARCO-TEJADA, P.J.; DEXTRAZE, L. Integration of narrow-band vegetation indices for prediction of crop chlorophyll content for application to precision agriculture. Remote Sensing of Environment, New York, v.81, n.2, p.41626, 2002.

HUDSON, W.D.; RAMM, C.W. Correct formulation of the kappa coefficient of agreement. Photogrammetric Engineering \& Remote Sensing, Falls Church, v.53, n.4, p.421-2, 1987.

KHATTREE, R.; NAIK, D.N. Multivariate data reduction and discrimination with SAS software. Cary: SAS Institute, 2000. 574 p.

MOGES, S.M.; RAUN, W.R.; MULLEN, R.W.; FREEMAN, K.W.; JOHNSON, G.V.; SOLIE, J.B. Evaluation of green, red and near infrared bands for predicting winter wheat biomass, nitrogen uptake, and final grain yield. Journal of Plant Nutrition, Monticello, v.27, n.8, p.1431-1441, 2004. 
ORLANDO, R.C.; PINTO, F.A.C.; QUEIROZ, D.M. de. Identificação automática de plantas daninhas e milho utilizando técnicas de visão artificial. In: CONGRESSO BRASILEIRO DE AGRICULTURA DE PRECISÃO, 1., 2004, São Pedro. Anais... 2004. 1 CD-ROM.

REGAZZI, A.J. Modelos de regressão não-linear e aplicações utilizando o SAS (PROC NLIN). 2.ed. Viçosa: UFV. 2000, 131 p.

ROSOLEM, C.A.; MARUBAYASHI, O.M. Seja o doutor do seu feijoeiro. Disponível em: <http://www.potafos.org/ppiweb/brazil.nsf/> Acesso em: 14 out. 2004.

SCHRÖDER, J.J.; NEETESON, J.J.; OENEMA, O.; STRUIK, P.C. Does the crop or the soil indicate how to save nitrogen in maize production? Reviewing the state of the art. Field Crops Research, Amsterdam, v.66, n.2, p.151-64, 2000.

SENA JÚNIOR, D.G. Utilização de técnicas de visão artificial para ajuste da adubação nitrogenada em trigo. 2005. 166 f. Dissertação (Mestrado em Máquinas Agrícolas) - Universidade Federal de Viçosa, Viçosa, 2005.

SÉRÉLÉ, C.Z.; GWYN, Q.H.J.; BOISVERT, J.B.; PATTEY, E.; MCLAUGHLIN, N.; DAOUST, G. Detection of corn nitrogen status from airborne vis-nir imagery using artificial neural networks. In: INTERNATIONAL CONFERENCE ON PRECISION AGRICULTURE, 5., 2000, Bloomington. Proceedings ... Madison: ASA-CSSA-SSSA, 2000. 1 CD-ROM.

SILVEIRA, P.M.; BRAZ, A.J.B.P.; DIDONET, A.D. Uso do clorofilômetro como indicador da necessidade de adubação nitrogenada em cobertura no feijoeiro. Pesquisa Agropecuária Brasileira, Brasília, v.38, n.9, p.1083-7, set. 2003.

VARELLA, C.A.A.; PINTO, F.A.C.; SANTOS, N.T.; QUEIROZ, D.M. Estimativa de estresse nutricional de nitrogênio em imagens digitais da cultura do milho. In: SIMPÓSIO

INTERNACIONAL DE AGRICULTURA DE PRECISÃO, 3., 2005, Sete Lagoas. Anais... 2005. 1 CD-ROM.

WOLFE, D.W.; HENDERSON, D.W.; HSIAO, T.C.; ALVINO, A. Interactive water and nitrogen effects on senescence of corn. II. Photosynthetic decline and longevity of individual leaves. Agronomy Journal, Madison, v.80, n.6, p.865-70, 1988. 